\title{
Influence of Preload and Nonlinearity of Railpads on Vibration of Railway Tracks under Stationary and Moving Harmonic Loads
}

\author{
Samuel G Koroma '* , Mohammed FM Hussein² and John S Owen ${ }^{3}$ \\ 'Institute of Sound and Vibration Research, University of Southampton, Southampton, \\ SOI7 IBJ, UK (Formerly at University of Nottingham) \\ ${ }^{2}$ Department of Civil and Architectural Engineering, Qatar University, P.O. Box 27I3, \\ Qatar (Formerly at University of Nottingham) \\ ${ }^{3}$ Department of Civil Engineering, University of Nottingham, Nottingham, NG7 2RD, UK
}

First submitted: 6 May 2014/Revised: 21 July 2014, 17 February 2015/Accepted: 20 February 2015

\begin{abstract}
In railway track dynamics, the stiffness and damping properties of railpads have a significant effect on track vibration, decay rates as well forces transmitted to the track supporting structure. Many studies have shown that railpads exhibit pronounced nonlinear behaviour, with preload and frequency dependent properties. This paper presents a three parameter railpad model, together with its differential equation of motion and the required model parameters obtained from experimental data. A time domain model of a rail discretely supported on these railpads is then formulated using the finite element method. The model is subjected to static and dynamic loading in order to study the effects of preload and frequency on its dynamic behaviour. Results are shown as time histories and frequency spectra for the track displacements and reaction forces for various preload levels. They emphasise the necessity of accounting for nonlinear behaviour based on the large disparities (up to $20 \mathrm{~dB}$ ) observed between the linear and nonlinear cases for the parameters used in this study.
\end{abstract}

\section{INTRODUCTION}

The type of railpad model adopted in modelling the dynamic behaviour of a railway track influences the accuracy of prediction of vibration of the track, as well as the forces transmitted through the railpad into the track supporting structure. Owing to the relative simplicity of linear analysis and the potential of obtaining exact analytical solutions, linear railpad properties have been widely assumed by many authors over the years [1-3]. However, many studies, including [4-7], have shown that railpads exhibit pronounced nonlinear behaviour and their properties are dependent on preload and frequency. Characterising these dependencies is, to a large extent, subjective to a particular data set and is by no means a generic process. It has been suggested by [8], that "a closer representation of the dynamic properties of a particular material can be achieved by devising a more complicated arrangement of spring and dashpot elements and, although the dynamic properties may be more accurately represented, the parameters required cannot be related to the fundamental physical properties of the material". Therefore, this exercise should be carried out with the scope set to particular applications, for e.g. in railway track dynamics, the scope may be to predict the vibration levels in a track for which experimental data is available for the railpads.

Some attempts have been made to characterise the nonlinear static and dynamic properties of railpads and rubber material in general. Examples include fractional

*Corresponding author: S.G.Koroma@soton.ac.uk (S G Koroma) 
derivative models $[9,10]$, the generalised Zener model [11]; the Poynting-Thomson model [6], etc. The generalised Zener model consists of a parallel array of an infinite number of standard linear solid models. The Poynting-Thomson model, on the other hand, consists of a frequency independent spring in parallel with a Maxwell combination of spring and dashpot in series. Reference [6] presented an empirical expression for the dynamic stiffness of this type of railpad model using curve fitting procedures. However, the expression was derived explicitly as a function of frequency, thereby limiting its use to single frequency problems; e.g. a linear single degree of freedom system excited by a harmonic load.

The modelling of a railway track with nonlinear railpads and ballast was presented in [12]. The individual nonlinear load-deflection laws for the railpad and ballast were combined into one load-deflection relationship for the whole track foundation, which was used to obtain the local preloads in the track foundation caused by a single wheel load. These were then used in a discretely supported track model to obtain the track receptances and decay rates. In a subsequent work [13], the calculation of receptances and decay rates of a similar track preloaded with multiple wheels was presented. These studies did not, however, consider the frequency dependent behaviour of railpads as well as the track dynamic behaviour under moving load conditions. The response of infinite beams on nonlinear elastic foundations subjected to harmonic loads has been studied by [14], [15], etc. using perturbation techniques. These studies utilised a two parameter nonlinear model for the elastic foundation, accounting only for load amplitude dependence. However, according to [16], a three parameter model such as the Poynting-Thomson model is necessary to account for both preload and frequency dependence of a railpad.

Other more recent studies in the area of railway track dynamics and ground vibration are as follows. A two-step approach for studying ground vibration due to railway operation is presented by [17]. The first step involves modelling the vehicletrack system including an approximate discrete soil layer, presented in [18], to calculate the track-ground interaction forces. These are then used as input for the second step, which is calculating ground vibration. In a subsequent paper, [19], a parametric study is conducted on the effects of vehicle and track properties on the level of ground vibration in the vicinity of the track. It is shown that the vibration level is not only affected by the vehicle types and speeds, but also by the railpad stiffness, rail mass and bending stiffness, sleeper mass and spacing. In [20] a comprehensive three dimensional time domain vehicle-track model excited by wheel flats is studied. Nonlinearity of the railpad and ballast are considered as exponential functions of their respective displacements. The results, plotted as peak wheel/rail contact forces show larger values for the linear track than for the non-linear one. This observation is inconsistent with [21], where stiffer foundations lead to larger contact forces.

In this paper, the static and dynamic properties of the railpad are modelled using a three parameter Poynting-Thomson (P-T) model. The differential equation of motion for this model is derived in section 2 , with the necessary model parameters obtained by curve fitting of experimental data presented in [5]. In Section 3.1, this railpad model is incorporated into a finite element based model of a railway track in order to study its effects on the track dynamic behaviour. The model is solved in the time domain using a time integration scheme for both stationary and moving load cases as described in Section 4. For the purpose of validating the results from this model, a semi-analytical solution is presented in Section 3.2 for linear parameters, using the Fourier Transformation Method. Results are then presented in Section 5 for the track displacements and reaction forces both as time histories and as frequency spectra for various levels of static load.

\section{RAILPAD STIFFNESS AND DAMPING PROPERTIES}

In this section, the static and dynamic load-displacement relationships of a railpad are presented. Based on experimental data presented in [5], empirical approximations are derived for both the static and dynamic stiffnesses of a railpad using a three parameter P-T visco-elastic model, see [6]. This model consists of a spring that is in parallel with a series combination of spring and dashpot. The latter 
two parameters ensure that the frequency dependence of the railpad is accounted for, as will be shown later from the differential equation of motion. In this model, the spring represents the stiffness and the dashpot the damping of the railpad. Section 2.1 deals with the static stiffness while Section 2.2 deals with the dynamic stiffness.

\section{I. Static stiffness of railpads}

The characterisation of a railpad's load-displacement relationship, and hence its static stiffness is presented in this section. For static loading conditions, the railpad is assumed to behave purely as an elastic material with nonlinear load-displacement relationship. Consider the static force, $p_{0}$, on the railpad is a nonlinear function of its static displacement, $u_{0}$, which can be described by a polynomial of degree, $\alpha$,

$$
p_{0}\left(u_{0}\right)=k_{1} u_{0}+k_{2} u_{0}^{2}+\ldots+k_{j} u_{0}^{j}+\ldots+k_{\alpha} u_{0}^{\alpha}
$$

where $k_{1}, \ldots, k_{\alpha}$ are the coefficients of the polynomial and are obtained through nonlinear curve fitting of experimental data, and $j$ is a positive integer. In order to ensure that the fit between $p_{0}$ and $u_{0}$ is well conditioned, the approximation is done with $p_{0}$ being in $\mathrm{kN}$ and $u_{0}$ in $\mathrm{mm}$.

From equation (1), two types of stiffness can be obtained; the secant stiffness, $k_{s}=\Delta p_{0} / \Delta u_{0}$, and the tangent stiffness, $k_{t}=d p_{0} / d u_{0}$. A seventh degree polynomial was found to sufficiently describe the $p_{0}-u_{0}$ relationship given in [5]. The values of the coefficients are $k_{1}=20.00 \mathrm{kN} / \mathrm{mm}, k_{3}=3.94 \times 10^{3} \mathrm{kN} / \mathrm{mm}^{3}, k_{5}=-1.78 \times 10^{3}$ $\mathrm{kN} / \mathrm{mm}^{5}$ and $k_{7}=3.2 \times 10^{3} \mathrm{kN} / \mathrm{mm}^{7}$, representing only stiffening load-deflection behaviour of the railpad. The coefficients of the polynomial that are not included here are zero. Note that when fully unloaded, the railpad possesses an unloaded stiffness of $k_{1}$.

Figure 1 shows the approximated nonlinear load-deflection and load-stiffness plots together with the corresponding data from [5], and confirms the good agreement between equation (1) and experimental data.

In the construction of railway tracks, each railpad is preloaded by clips that hold it in place and by the weight of the rail that is supported within one fastener bay (i.e. $0.6 \mathrm{~m}$ length of rail). The total initial preload on the railpad was calculated by [12] to be about $20.36 \mathrm{kN}$ with $60 \mathrm{E} 1$ type rail. To account for this initial preload, the reaction force in the railpad due to external track loads are added to this preload. Also, it is assumed that the railpad is in static equilibrium at this preload, and all displacements are taken with reference to this point.

\subsection{Dynamic stiffness and damping of railpads}

In addition to the dependence of railpad stiffness on preload as outlined in the previous section, the stiffness is also dependent on frequency. Some data of dynamic stiffness obtained from laboratory measurements have been presented by $[4,5,7]$ for studded rubber railpads that are commonly used in European railways. In [5], the dynamic stiffness values are given for five preload levels: 20, 30, 40, 60, and $80 \mathrm{kN}$, over a frequency range of $40-1000 \mathrm{~Hz}$. Using this data, approximations of the

(a)

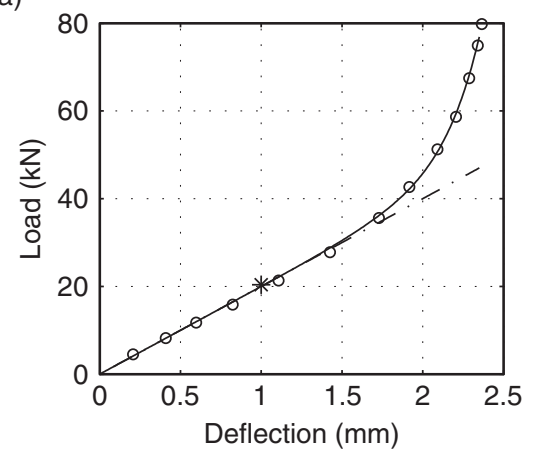

(b)

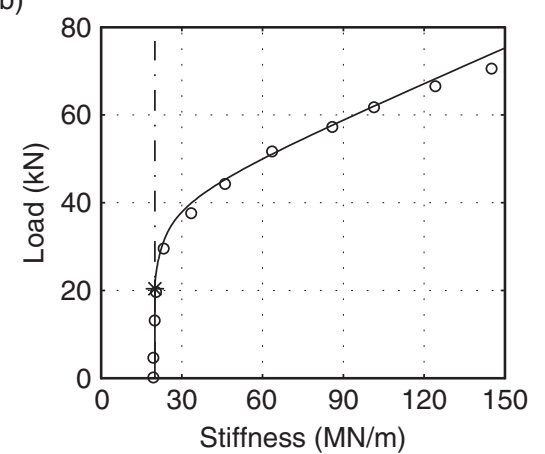

Figure I. Static load-deflection and load-stiffness plots for Pandrol $10 \mathrm{~mm}$ studded rubber railpad. $-\cdot-$ : linear, — 


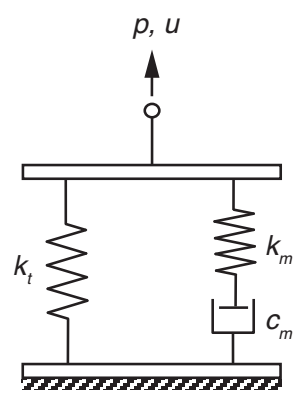

Figure 2. Poynting-Thomson model for a railpad with nonlinear elastic spring of stiffness $k_{t}$ in parallel with a series combination of spring of stiffness $k_{m}$ and dashpot of damping factor $c_{m}$

dynamic stiffness as a function of preload and loading rate are derived using nonlinear curve fitting.

Figure 2 shows a Poynting-Thompson railpad model subjected to a force, $p$, with a corresponding displacement, $u$. The P-T model is analysed in two parts: the frequency independent spring part and the Maxwell part consisting of the spring and dashpot in series. The series arrangement of the the Maxwell part means that the reaction force, $p_{m}$, in the spring and dashpot is the same, but the total displacement, $u_{m}$, is the sum of the displacements experienced by the two components. Thus

$$
\begin{gathered}
p_{m}=k_{m} u_{s p}=c_{m} \dot{u}_{d p} \\
u_{m}=u_{s p}+u_{d p}
\end{gathered}
$$

where the subscripts 'sp' and 'dp' represent 'spring' and 'dashpot' respectively.

In order to obtain one governing equation relating $p_{m}$ and $u_{m}$, it is necessary to differentiate equations (2a) and (2b) with respect to time, and substitute for $\dot{u}_{s p}$ and $\dot{u}_{d p}$ from equation $(2 \mathrm{a})$. This results in

$$
\dot{p}_{m}+\frac{k_{m}}{c_{m}} p_{m}=k_{m} \dot{u}_{m}
$$

Considering the parallel arrangement of the frequency independent spring and the Maxwell part, the displacement is the same for the two parts, but the total force is the sum of the individual forces in the two parts. Thus

$$
\begin{aligned}
& p=p_{t}+p_{m}, \\
& u=u_{t}=u_{m},
\end{aligned}
$$

where the force $p_{t}$ is a nonlinear function of the static part of the total displacement $u$.

Again, to obtain one differential equation for the whole P-T model, equation (4a) is differentiated with respect to time as

$$
\dot{p}=\frac{d p_{t}}{d u} \dot{u}+\dot{p}_{m}
$$

and by substituting for $p_{m}$ and $\dot{p}_{m}$ from equation (4a) and equation (5) respectively into equation (3), one obtains

$$
\dot{p}+\frac{k_{m}}{c_{m}} p=\left(k_{m}+\frac{d p_{t}}{d u}\right) \dot{u}+\frac{k_{m}}{c_{m}} p_{t}
$$

Equation (6) is the differential equation that describes the dynamic behaviour of the P-T model and can be seen to contain a loading rate term that captures the frequency dependent behaviour of the railpad.

Consider that the force, $p$, is composed of a static preload, $p_{0}$, and a dynamic load, $p_{d y n}$. The resulting tangent stiffness due to the preload is $k_{t}=d p_{t} / d u_{0}$, due to the 
nonlinear $p_{0}-u_{0}$ relationship. Assuming that the dynamic displacement of the railpad is in linear relation with the dynamic load acting on it, the dynamic part of $p_{t}$ is then $k_{t} u_{d y n}$, where $u_{d y n}$ is the dynamic displacement. Now, if $p_{d y n}$ varies harmonically, then the resulting dynamic stiffness of the railpad can be shown to be

$$
k_{d y n}=k_{t}\left[1+\frac{k_{m}}{k_{t}} \frac{\mathrm{i} \omega_{0} \tau}{1+\mathrm{i} \omega_{0} \tau}\right]
$$

where $\tau=c_{m} / k_{m}$ is the relaxation time of the railpad. The parameters $k_{m}$ and $c_{m}$ are obtained from nonlinear curve fitting. The values obtained for $k_{m}$ and $c_{m}$ from the optimisation show some preload dependence, in accordance with the following equations

$$
\begin{aligned}
& k_{m}=k_{m 0}\left(1+\beta^{r}\right) \\
& c_{m}=c_{m 0}\left(1+\beta^{s}\right)
\end{aligned}
$$

where $k_{m 0}$ and $c_{m 0}$ are the respective values of $k_{m}$ and $c_{m}$ at a reference preload value, $p_{c} ; k_{m 0}=60.33 \times 10^{6} \mathrm{~N} / \mathrm{m}$ and $c_{m 0}=22.5 \times 10^{4} \mathrm{~N} . \mathrm{s} / \mathrm{m} ; \beta$ is a ratio of the preload increment relative to $p_{c}$, i.e. $\beta=\left(p-p_{c}\right) / p_{c} . r$ and $s$ are the exponential preload influences and are almost identical $(r=1.9954$ and $s=2.07)$, but for simplicity a value of 2 is used for both of them. The reference preload is taken as $20.36 \mathrm{kN}$, i.e. the initial preload.

Approximations of $k_{d y n}$ in equation (7) for the P-T model, together with the optimised parameters, are plotted in Figure 3 against frequency for five preload levels. It can be seen in Figure 3 that there is a good agreement between equation (7) and the experimental data. Although experimental data is not available between $0-40 \mathrm{~Hz}$, it is assumed for the P-T model that the dynamic stiffness increases gradually from a value of $k_{t}$ at $0 \mathrm{~Hz}$ to its value at $40 \mathrm{~Hz}$, disregarding any resonance that may be present within this range.

In the next section, the P-T railpad model is incorporated into a model of a railway track to study its effect on railway track dynamics.

\section{MODELLING OF TRACK DYNAMICS}

In this section, two models of a railway track are presented. The first, described in Section 3.1, is a time domain model of a discretely supported rail, formulated using the Finite Element Method (FEM). This model is used to study the effect of nonlinear railpad properties on track dynamics. The second model is presented in Section 3.2 and is solved using the Fourier Transformation Method (FTM).

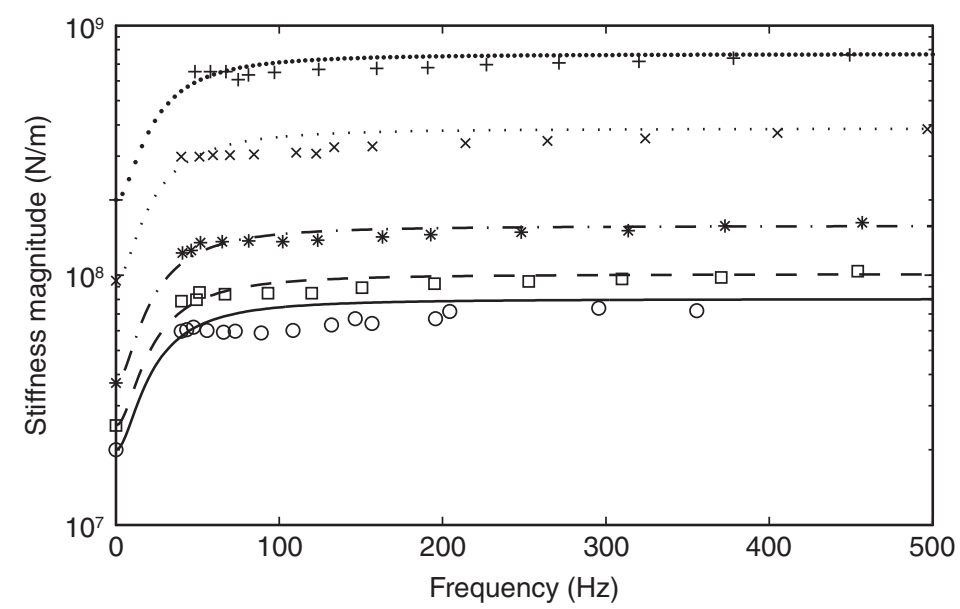

Figure 3. Approximated railpad dynamic stiffness plotted against frequency for five preload levels (lines) compared with data points from [5] (markers). $\longrightarrow, \circ: 20 ;---, \square: 30 ;-\cdot-, *: 40 ; \ldots . ., \times: 60$ and ....., $+: 80 \mathrm{kN}$ 


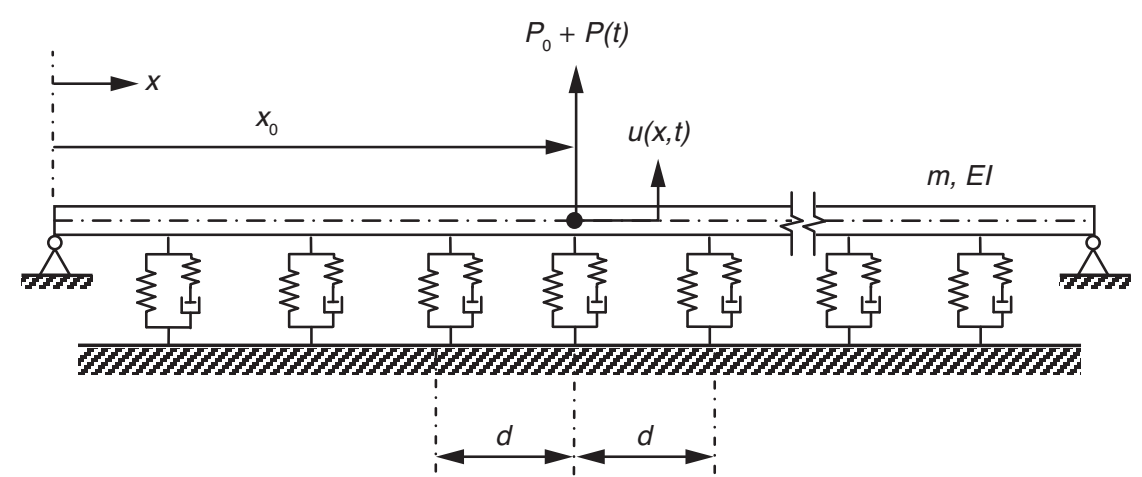

Figure 4. Rail discretely supported on a three parameter P-T elastic foundation and subjected to combined static and dynamic load.

This model uses linear railpad properties and is used to validate the FEM for linear parameters.

\section{I. Nonlinear time domain track model}

The P-T railpad model formulated in Section 2 will be used in a finite element (FE) based model of a railway track in order to investigate the effects of preload and frequency dependence of railpads on the dynamic behaviour of a railway track. The model description and formulation of the equations of motion as well as the solution method will be discussed in the following sections.

\section{I.I. Model description}

Figure 4 shows a model of a railway track. The rail is modelled as an EulerBernoulli beam with bending stiffness, EI, and mass per unit length, $m$, discretelysupported on railpads at regular intervals, $d$, and is simply supported at its ends. The railpads are modelled using the three parameter P-T model discussed in the previous section. For the purpose of studying only the effects of railpad properties on the dynamic behaviour of the track, it is assumed that the forces transmitted through the railpads are mainly controlled by the rail-railpad system, for cases where the railpad supporting structure is much stiffer than the railpad, and can therefore be considered rigid.

\section{I.2. Discretisation of the beam and system matrices}

The rail is discretised into $N$ finite elements, each having two nodes and four degreesof-freedom (DOF), i.e. vertical translation and rotation at each node. The length of each of these elements, $L$, is chosen such that $d / L=q$, where $q$ is a positive integer. Let the number of railpad bays in the track model be $n$, so that the number of railpads, $N_{R}=n+1$. The total number of elements in the track structure is therefore $N=n \times q$, the number of nodes is $N+1$ and the total number of DOF $N_{D}=2(N+1)$.

The stiffness and consistent mass matrices and the external nodal force vector for the ith beam element are denoted by $\mathbf{K}_{B, i}, \mathbf{M}_{i}$ and $\mathbf{P}_{i}$ respectively. Expressions for these are readily available in the literature, e.g. [22]. The global stiffness and mass matrices of the rail, $\mathbf{K}_{B}$ and $\mathbf{M}$ respectively, are assembled using compatibility conditions at the nodes, i.e. displacement, slope and bending moments are continuous. In this way, the local matrix and vector components corresponding to the mutual nodes between two consecutive elements are overlapped.

In addition to the global mass and stiffness matrices for the rail/beam, global stiffness and damping matrices for the railpads should be formed and coupled to the beam in order to obtain global matrices of the track structure. Coupling of the beam to the railpads and formulation of the equation of motion for the entire track structure are carried out in the next section.

\section{I.3. Coupled equation of motion of the track}

The coupling of a continuous rail/beam to equally spaced railpads is shown in Figure 5. The beam is acted upon by external nodal forces contained in the vector, $\mathbf{P}$, 


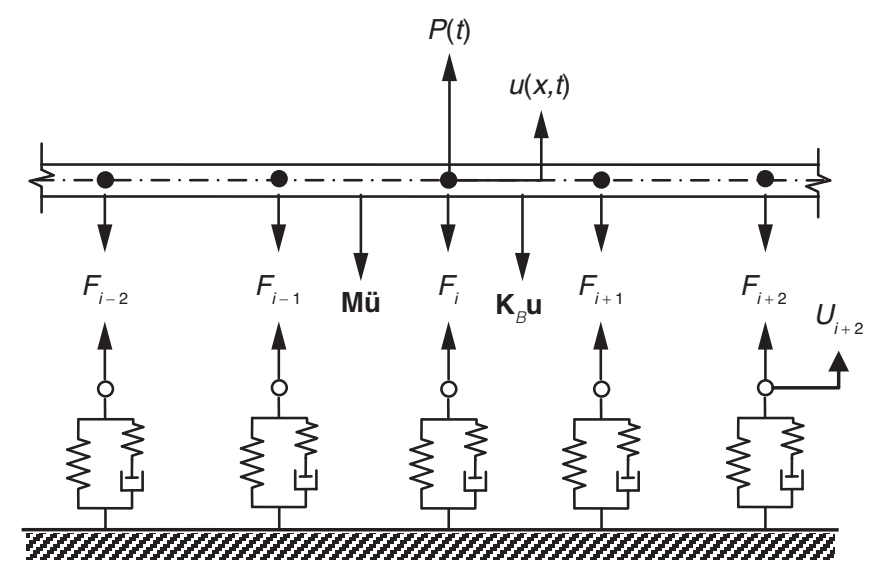

Figure 5. Coupling of the beam to the P-T railpad models.

resulting in inertial and reaction forces due to its mass and bending stiffness, i.e. Mü and $\mathbf{K}_{B} \mathbf{u}$ respectively. Consider that additional reaction forces, $F_{i}$, for $i=1, \ldots, N_{D}$, are present in the coupled structure due to the presence of the railpads. These forces, which are contained in a global force vector, F, are non-zeros only at the vertical DOF of the nodes at which the railpads are located.

The differential equations of motion of the beam and the railpads are given by

$$
\begin{gathered}
\mathbf{M u ̈}+\mathbf{K}_{B} \mathbf{u}+\mathbf{F}=\mathbf{P} \\
{\left[\mathbf{K}_{M}+\mathbf{K}_{T}\right] \dot{\mathbf{u}}_{P}+\frac{1}{\tau} \mathbf{K}_{T} \mathbf{u}_{P}=\frac{1}{\tau} \mathbf{F}_{P}+\dot{\mathbf{F}}_{P}}
\end{gathered}
$$

where $\mathbf{u}_{P}$ and $\mathbf{F}_{P}$ are $N_{R} \times 1$ vectors that contain the vertical displacements and reaction forces of the nodes at which the railpads are located, therefore they are subsets of the global beam displacement and reaction force vectors, $\mathbf{u}$ and $\mathbf{F}$ respectively. $\mathbf{K}_{M}$ and $\mathbf{K}_{T}$ are $N_{R} \times N_{R}$ diagonal matrices containing the $k_{m j}$ and $k_{t j}$ values of the railpads respectively, for $j=1, \ldots, N_{R}$. The relaxation time $\tau=c_{m 0} / k_{m 0}$, since the preload influence for both the stiffness and damping of the Maxwell branch is the same.

The presence of the load rate, $\dot{\mathbf{F}}_{P}$, in equation ( $9 \mathrm{~b}$ ) prevents a simple substitution of the railpad matrices into the beam matrices. Therefore, the coupling is sought by making $\mathbf{F}_{P}$ part of the global unknown vector of the track system. By assembling equations (9a) and (9b) into matrix form, the coupled system equation can be formed as follows

$$
\left[\begin{array}{cc}
\mathbf{M} & \mathbf{0} \\
\mathbf{0} & \mathbf{0}
\end{array}\right]\left\{\begin{array}{c}
\ddot{\mathbf{u}} \\
\ddot{\mathbf{F}}_{P}
\end{array}\right\}+\left[\begin{array}{cc}
\mathbf{0} & \mathbf{0} \\
\mathbf{A} & -\mathbf{I}
\end{array}\right]\left\{\begin{array}{c}
\dot{\mathbf{u}} \\
\dot{\mathbf{F}}_{P}
\end{array}\right\}+\left[\begin{array}{cc}
\mathbf{K}_{B} & \mathbf{J} \\
\frac{1}{\tau} \mathbf{B} & -\frac{1}{\tau} \mathbf{I}
\end{array}\right]\left\{\begin{array}{c}
\mathbf{u} \\
\mathbf{F}_{P}
\end{array}\right\}=\left\{\begin{array}{c}
\mathbf{P} \\
\mathbf{0}
\end{array}\right\}
$$

The coupled system in equation (10) has the same number of DOF, $N_{D}$, but the number of unknowns is $N_{D}+N_{R}$. The sub matrices are defined as follows: $\mathbf{A}$ and $\mathbf{B}$ are $N_{R} \times N_{D}$ matrix with non-zero elements $\mathbf{A}_{j, 2 q(j-1)+1}=\mathbf{K}_{M_{j, j}}+\mathbf{K}_{T_{j, j}}$, and $\mathbf{B}_{j, 2 q(j-1)+1}=\mathbf{K}_{T_{j, j}} . \mathbf{J}$ is a $N_{D} \times N_{R}$ matrix that contains unit values only at the DOF corresponding to the positions of the railpads, i.e. $\mathbf{J}_{2 q(j-1)+1, j}=1$, and $\mathbf{I}$ is an identity matrix of size $N_{R} \times N_{R}$.

\section{I.4. Solution of the equation of motion of the track}

Solution of the differential equations of motion for the FE model is a two stage process. First, the preloads, and hence the preloaded stiffnesses of the railpads are calculated based on the static $p_{0}-u_{0}$ relationship and according to the magnitude of the static load. These are then used as input to study the dynamic behaviour of the 
track. The first part will depend on whether the load is stationary or moving and will therefore be discussed later in Section 4 .

Once the preloaded stiffnesses have been obtained, the differential equation is solved only for the dynamic loading, with the complete solution being a sum of the static and dynamic parts. The solution is obtained using a two sub-step implicit time integration scheme [23].

\subsection{Solution by the Fourier Transformation Method}

The model described in Section 3.1 can also be solved, for the linear case, using the FTM in the wavenumber-frequency domain. For simplicity, consider an infinite rail continuously supported on a P-T railpad model with distributed stiffness, $\bar{k}_{t}=k_{t} / d$ and $\bar{k}_{m}=k_{m} / d$, and damping $\bar{c}_{m}=c_{m} / d$. The rail is subjected to an oscillating load, $P_{1} \mathrm{e}^{\mathrm{i} \omega_{0} t}$, moving at speed $v$, such that it arrives at position $x=0$ at time $t=0$.

The partial differential equation of motion governing the dynamic behaviour of the rail is

$$
E I \frac{\partial^{4} u(x, t)}{\partial x^{4}}+m \frac{\partial^{2} u(x, t)}{\partial t^{2}}+F(x, t)=P_{1} \mathrm{e}^{\mathrm{i} \omega_{0} t} \delta(x-v t),
$$

and that for the railpad is

$$
\frac{\partial F(x, t)}{\partial t}+\frac{1}{\tau} F(x, t)=\left(\bar{k}_{m}+\bar{k}_{t}\right) \frac{\partial u(x, t)}{\partial t}+\frac{1}{\tau} \bar{k}_{t} u(x, t)
$$

where $u(x, t)$ is the displacement of the rail at any point, $x$, at an instant, $t$, and $F(x, t)$ is distributed reaction force in the railpad.

The solution of equations (11) and (12) is obtained using the FTM. For a function in the space-time domain, $g(x, t)$, the Double Fourier transform pair relating it to its counterpart in the wavenumber-frequency domain, $\tilde{g}(\xi, \omega)$, can be given as

$$
\begin{aligned}
& g(x, t)=\frac{1}{4 \pi^{2}} \int_{-\infty}^{\infty} \int_{-\infty}^{\infty} \tilde{g}(\xi, \omega) \mathrm{e}^{\mathrm{i} \xi x} \mathrm{e}^{\mathrm{i} \omega t} \mathrm{~d} \xi \mathrm{d} \omega \text { and } \\
& \tilde{g}(\xi, \omega)=\int_{-\infty}^{\infty} \int_{-\infty}^{\infty} g(x, t) \mathrm{e}^{-\mathrm{i} \xi x} \mathrm{e}^{-\mathrm{i} \omega t} \mathrm{~d} x \mathrm{~d} t,
\end{aligned}
$$

where $\xi$ and $\omega$ are the wavenumber and angular frequency of each harmonic component of $g$.

Applying equation (13) on equations (11)-(12) from the $x-t$ domain to the $\xi-\omega$ domain, results in

$$
\left(E I \xi^{4}-m \omega^{2}\right) \tilde{u}(\xi, \omega)+\tilde{F}(\xi, \omega)=2 \pi P_{1} \delta\left(\omega-\omega_{0}+\xi v\right),
$$

and

$$
(1+\mathrm{i} \omega \tau) \tilde{F}(\xi, \omega)=\left(\bar{k}_{t}+\left(\bar{k}_{m}+\bar{k}_{t}\right) \mathrm{i} \omega \tau\right) \tilde{u}(\xi, \omega) .
$$

Substituting for $\widetilde{F}(\xi, \omega)$ from equation (15) into equation (14) gives the solution of the rail displacement in the $\xi-\omega$ domain as

$$
\tilde{u}(\xi, \omega)=\frac{2 \pi P_{1} \delta\left(\omega-\omega_{0}+\xi v\right)}{E I \xi^{4}-m \omega^{2}+\bar{k}_{t}\left(1+\frac{\bar{k}_{m}}{\bar{k}_{t}} \frac{\mathrm{i} \omega \tau}{1+\mathrm{i} \omega \tau}\right)} .
$$

The response in the space-time domain can be obtained, using the inverse double Fourier transformation, as

$$
\tilde{u}(x, t)=\frac{P_{1} \mathrm{e}^{\mathrm{i} \omega_{0} t}}{2 \pi} \int_{-\infty}^{\infty} \frac{\mathrm{e}^{\mathrm{i} \xi(x-v t)}}{E I \xi^{4}-m\left(\omega_{0}-\xi v\right)^{2}+\bar{k}_{t}\left(1+\frac{\bar{k}_{m}}{\bar{k}_{t}} \frac{\mathrm{i}\left(\omega_{0}-\xi v\right) \tau}{1+\mathrm{i}\left(\omega_{0}-\xi v\right) \tau}\right)} \mathrm{d} \xi
$$

and can be computed numerically. 


\section{APPLICATIONS}

In this section, the dynamic behaviour of the FE track model will be investigated under the action of combined static and dynamic loading. The case of a stationary harmonic load is described in Section 4.1 whilst Section 4.2 focuses on the moving load case.

\section{I. Stationary harmonic load}

The track is subjected to a non-moving load, $P_{t o t}(t)$ placed at a fixed point, a distance of $x_{0}$ from the left end of the track. The load consists of a static component, $P_{0}$, and a dynamic component, $P(t)$, where $P(t)$ is defined as a harmonic function with amplitude, $P_{1}$, and angular frequency, $\omega_{0}=2 \pi f_{0}, f_{0}$ being the excitation frequency. Hence, the load on the track can be described by

$$
P_{t o t}(t)=\left[P_{0}+P_{1} \mathrm{e}^{\mathrm{i} \omega_{0} t}\right] \delta\left(x-x_{0}\right)
$$

where $x_{0}=N L / 2$ corresponds to the middle node of the beam, directly on a railpad. Now $P_{t o t}(t)$ is placed in the global force vector, $\mathbf{P}$, at the corresponding vertical DOF of the middle node.

As mentioned earlier, the solution of this problem requires that the preloads, and hence the preloaded stiffnesses are first calculated before the dynamic behaviour can be investigated. To obtain the preloaded stiffnesses of the railpads under the effect of the static load, the following nonlinear static equation needs to be solved

$$
\mathbf{F}\left(\mathbf{u}_{0}\right)=\mathbf{P}_{0}
$$

where $\mathbf{F}\left(\mathbf{u}_{0}\right)$ is the nonlinear reaction force vector; i.e. $\mathbf{F}\left(\mathbf{u}_{0}\right)=\mathbf{K}\left(\mathbf{u}_{0}\right) \mathbf{u}_{0}$, with $\mathbf{K}\left(\mathbf{u}_{0}\right)$ being the nonlinear static stiffness as a function of the static displacements $\mathbf{u}_{0} . \mathbf{P}_{0}$ is the external nodal force vector consisting of $P_{0}$ as the only non-zero element, at the corresponding DOF where the external load is applied.

A Newton-Raphson iterative procedure is adopted to obtain the static displacements of the pads, and hence the preloaded stiffnesses. This procedure is summarised as

$$
\begin{gathered}
\mathbf{K}^{(i-1)} \Delta \mathbf{u}_{0}^{(t)}=\mathbf{P}_{0}-\mathbf{F}^{(i-1)} \\
\mathbf{u}_{0}^{(i)}=\mathbf{u}_{0}^{(i-1)}+\Delta \mathbf{u}_{0}^{(i)}
\end{gathered}
$$

where $\mathbf{K}^{(i-1)}$ and $\mathbf{F}^{(i-1)}$ are respectively the consistent tangent stiffness matrix and reaction force vector computed at the configuration corresponding to the static displacement, $\mathbf{u}_{0}^{(i-1)}$, for $i$ being the number of iterations. Convergence of equation (20) is achieved by satisfying a predefined convergence criterion that guarantees the degree of accuracy required for the solution.

Table 1 contains the static preloads for the case when a static load is positioned directly over a railpad. Due to symmetry, only the railpads on one side of the load have been presented.

It can be seen from Table 1 that when there is no load on the track, all pad preloads are equal to the initial value of $20.36 \mathrm{kN}$. They then increase for higher levels of static load and beyond a distance of about 3-4 $\mathrm{m}$ from the load, the pads are almost insensitive to load and remain fairly unloaded.

\subsection{Moving harmonic load}

Consider the FE track model is subjected to the same load combination as in Section 4.1, moving along the length of the beam with speed, $v$, in the positive $x$-direction. It is assumed that the track section considered (i.e. $0 \leq x \leq N L$ ) is initially at rest up to the arrival of the load at $x=0$ at time $t=0$ and that it returns back to rest after the load leaves the track section for $x>N L$. The total load moving on the track, $P_{t o t}(\mathrm{x}, t)$, can be described by

$$
P_{t o t}(x, t)=\left[P_{0}+P_{1} \mathrm{e}^{\mathrm{i} \omega_{0} t}\right] \delta(x-v t),
$$

where $x=v t$ corresponds to the position of the load at any instant, $t$. Now $P_{t o t}(\mathrm{x}, t)$ is converted into equivalent nodal forces of the element on which it acts using the Hermitian interpolation functions, see [24]. 

Influence of Preload and Nonlinearity of Railpads on Vibration of Railway Tracks
under Stationary and Moving Harmonic Loads

Table I.

Preloads (in kN) on the railpads in the vicinity of the static load

\begin{tabular}{lcccccc}
\multirow{2}{*}{$\begin{array}{l}\text { Pad position } \\
\text { from load }(\mathrm{m})\end{array}$} & 0.00 & 25.00 & 50.00 & 75.00 & 100.00 & 125.00 \\
\hline 0.00 & 20.36 & 28.47 & 37.26 & 48.24 & 62.57 & 79.40 \\
0.60 & 20.36 & 26.26 & 32.09 & 37.41 & 41.75 & 45.29 \\
1.20 & 20.36 & 23.08 & 25.56 & 27.50 & 28.73 & 29.49 \\
1.80 & 20.36 & 21.04 & 21.62 & 22.03 & 22.24 & 22.34 \\
2.40 & 20.36 & 20.18 & 20.00 & 19.83 & 19.69 & 19.58 \\
3.00 & 20.36 & 20.02 & 19.70 & 19.45 & 19.28 & 19.16 \\
3.60 & 20.36 & 20.12 & 19.91 & 19.74 & 19.63 & 19.56 \\
$\cdot$ &. &. &. &. &. &. \\
$\infty$ & & & initial preload of 20.36 & &
\end{tabular}

Under the action of the moving load, the static part of the load induces a loaded stiffness variation that changes with the position of the load. Thus, the preloaded stiffness distribution is time dependent and should be calculated with the history of the load's position taken into account. The stiffness distribution, as well as its rate of change, are dependent on the speed of the load. As a result, the use of the equilibrium conditions described by equation (19) may be restricted, since the quasi-static behaviour, which becomes increasingly significant as the speed of the load increases, should be considered. However, the lack of experimental data to describe the responses of railpads under quasi-static loading conditions presents a difficulty in obtaining the necessary parameters for characterising the dynamic (or quasi-dynamic) stiffness of the pad due to this loading condition. Therefore, two fundamental assumptions are adopted when considering moving loads on the nonlinear track model. These are:

1. For slowly moving loads relative to the critical velocity of the track, it can be assumed that the response of each railpad is static, therefore the static loaddeflection relationship remains valid. This assumption holds since the rate of loading and unloading of each railpad, as the load approaches and departs from its location, is not very rapid. Hence the static response of the pads due to a moving load is used to obtain the preloaded stiffnesses without including inertia or damping effects.

2. The effects of the static and dynamic loads can be separated from one another. This means that, at each time step, and hence each position of the load, the static load induces the preloaded stiffness variation of the railpads based on their static displacements, from which the dynamic stiffness parameters can be calculated. The dynamic load is then applied to the track to study the dynamic response.

In order to check the validity of the the first assumption, the following equation is used to calculate the quasi-static displacements of the track

$$
\mathbf{M} \ddot{\mathbf{u}}_{Q}(t)+\mathbf{K}\left(\mathbf{u}_{Q}, t\right) \mathbf{u}_{Q}(t)=\mathbf{P}_{0}
$$

which are used to obtain the preloaded stiffnesses, where the subscript "Q" represents the quasi-static part of the track response. These are then compared with those obtained from equation (19) for various load speeds. Equation (22) is solved using the composite implicit time integration scheme [23].

Table 2 shows the preloaded stiffness distributions of the railpads in the vicinity of a load moving at $v=0,50$ and $250 \mathrm{~ms}^{-1}$. For this case, the railpads' positions have been measured such that the one at $x=N L / 2$ is considered to be the origin, and is 
Table 2.

Preloaded stiffnesses (in $\mathrm{MN} / \mathrm{m}$ ) of the railpads in the vicinity of the static load of $75 \mathrm{kN}$ moving at 0,50 and $250 \mathrm{~ms}^{-1}$

\begin{tabular}{lccc} 
Pad position & \multicolumn{3}{c}{ Load speed $\left(\mathrm{ms}^{-1}\right)$} \\
\cline { 2 - 4 } from load $(\mathrm{m})$ & 0 & 50 & 250 \\
\hline$-\infty$ & 20.00 & unloaded stiffness of 20.00 & $\cdot$ \\
-2.40 & 20.08 & $\cdot$ & 20.00 \\
-1.80 & 21.37 & 20.00 & 20.08 \\
-1.20 & 28.80 & 21.56 & 21.68 \\
-0.60 & 53.56 & 29.94 & 32.23 \\
0.00 & 28.80 & 53.59 & 61.28 \\
0.60 & 21.37 & 27.84 & 27.99 \\
1.20 & 20.08 & 21.19 & 20.95 \\
1.80 & 20.00 & 20.06 & 20.00 \\
2.40 &. & 20.00 & 20.00 \\
. & &. &. \\
$\infty$ & & unloaded stiffness of 20.00 & \\
\hline
\end{tabular}

also the position where the load acts for the stationary load case. The variations are snapshots taken at the time when the moving load is directly at this origin. Note that due to convection of the load, the symmetry is altered and therefore railpads on either side of the load have been presented.

It can be seen that the stiffness distribution of the railpads does not drastically change for the range of load speeds shown, changing only by $3.96 \%$ up to $50 \mathrm{~ms}^{-1}$ and by $14.41 \%$ for speeds up to $250 \mathrm{~ms}^{-1}$. The length of the loaded zone, and hence extent of the loaded stiffnesses, remain fairly constant at around $4.5-6 \mathrm{~m}$.

Due to the bending stiffness of the rail, there is some negative deflection of the railpad relative to the equilibrium position induced by the fastener clips. This negative deflection leads to a reduction in the pad preload, but the railpad does not become fully unloaded since these displacements are small. Also, from Figure 1, it can be seen that the stiffness is constant up to about $20 \mathrm{kN}$, so that this reduction in preload below that applied by the clips does not lead to a reduction in the pad stiffness.

The model presented in Sections 3.1 and 4 will be validated, for linear parameters, using the solution obtained by the FTM.

\section{RESULTS AND DISCUSSION}

In this section, results will be presented for the FE track model subjected to stationary and moving harmonic loads. The effects of accounting for the preload and frequency dependence is studied using the P-T railpad model. Results for the stationary case are presented in Section 5.1, and the moving load results are dealt with in Section 5.2.

The following parameters are used in the numerical examples presented in this section: mass of rail, $m=60.21 \mathrm{~kg} / \mathrm{m}$, bending stiffness of rail, $E I=6.4 \mathrm{MN} \mathrm{m}^{2}$, unloaded railpad stiffness, $k_{1}=20 \mathrm{MN} / \mathrm{m}$, railpad spacing for the discretely supported case, $d=0.6 \mathrm{~m}$.

\section{I. Results for the stationary load}

The validity of the FEM is first checked against the FTM in Section 5.1.1 using linear parameters. In Section 5.1.2, results for the nonlinear FEM are presented to demonstrate the effects of preload on railway track dynamics. 
Influence of Preload and Nonlinearity of Railpads on Vibration of Railway Tracks under Stationary and Moving Harmonic Loads
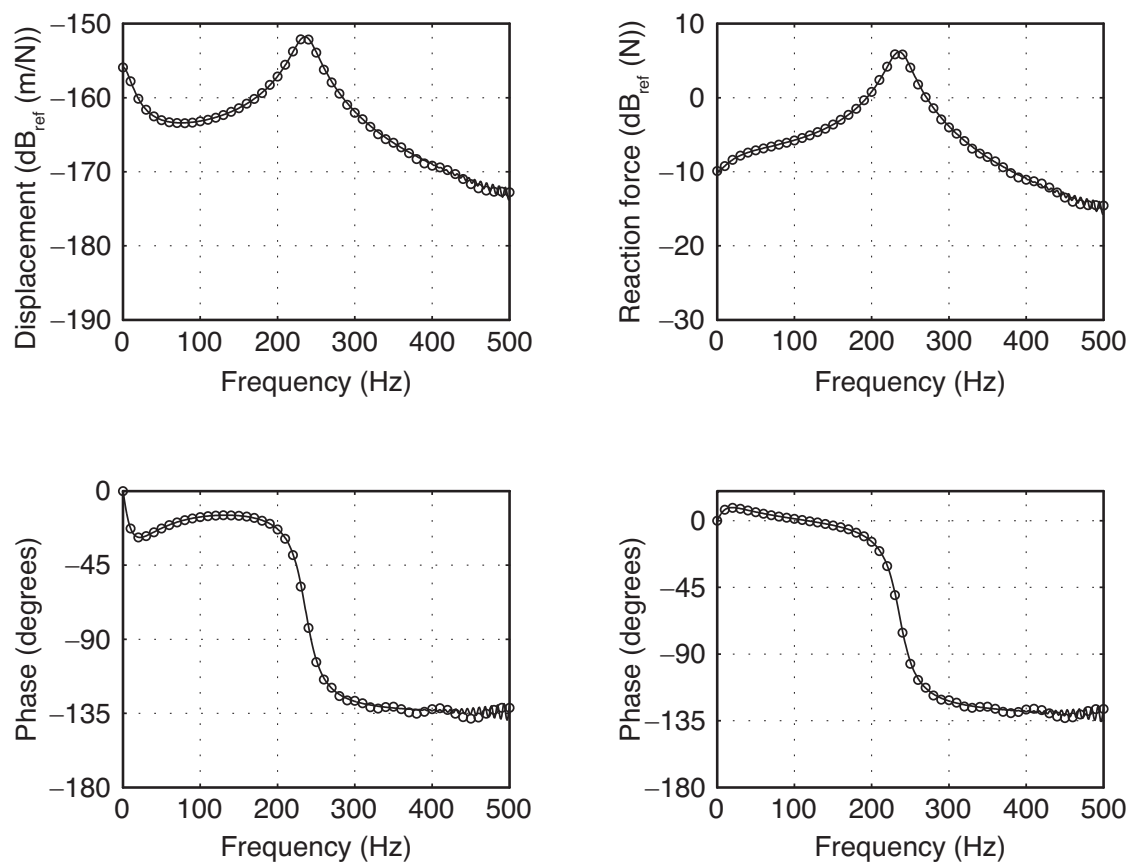

Figure 6. Steady-state magnitude and phase of $(a-b)$ the displacement, $(c-d)$ the reaction force, at a point under the load plotted against excitation frequency. Comparison between the FTM (—) and FEM (०) for linear parameters.

\section{I.I. Validation of the FE model for linear parameters}

The FTM is used to validate the results obtained from the FEM for the linear case, which is achieved by setting $P_{0}=0$ in the latter. In the FEM, 320 elements of length $0.3 \mathrm{~m}$ are used with a time step of $T / 50$, where $T$ is the period of oscillation. In the FTM, on the other hand, 2048 wavenumber samples are used to compute equation (17), with $x=0$ and $v=0$.

Figure 6 shows the dynamic displacement and reaction force amplitudes and phase plotted as a function of excitation frequency for a point that is directly under the load. It is apparent that the two models are in good agreement. In Fig. 6(a), the displacement can be seen to reduce by as much as $7.5 \mathrm{~dB}$ from its value at $0 \mathrm{~Hz}$ up to a frequency of $80-100 \mathrm{~Hz}$. At this stage the dynamic stiffness of the railpad increases sharply, at a higher rate than the dynamic reaction force on the railpad, causing a reduction in the response. This can also be observed on the phase plot in Fig. 6(b). However, as the frequency increases, the inertial force from the rail becomes increasingly dominant, leading to an increase in the response up until the cut-on frequency, before gradually decaying beyond this point. The cut-on frequency occurs at $f_{c o} \approx(1 / 2 \pi) \sqrt{\left(k_{t}+k_{m}\right) / m d} \approx 237 \mathrm{~Hz}$. The corresponding amplitude and phase angle of the reaction force in the railpad are plotted against frequency in Figs. 6(c) and 6(d). It shows an increase in the force with frequency up until the cut-on frequency, before gradually reducing beyond this point.

\subsubsection{Effect of preload}

Results are now presented for the nonlinear FE model subjected to stationary load in order to study the effects of preload and frequency on railway track dynamics. Five levels of static load are considered: 0, 25, 50, 75 and $100 \mathrm{kN}$. Superimposed on each of these is a unit amplitude dynamic load. The preloaded stiffness distribution is calculated using equation (20) and then used as input to study the dynamic response.

Figure 7 shows the dynamic displacement and reaction force amplitudes and phase plotted as a function of excitation frequency for a point that is directly under the load. Comparison for various preload levels is shown. In Fig. 7(a), the response 
(a)

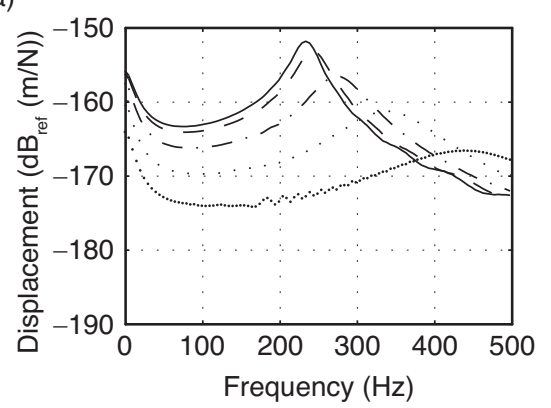

(b)

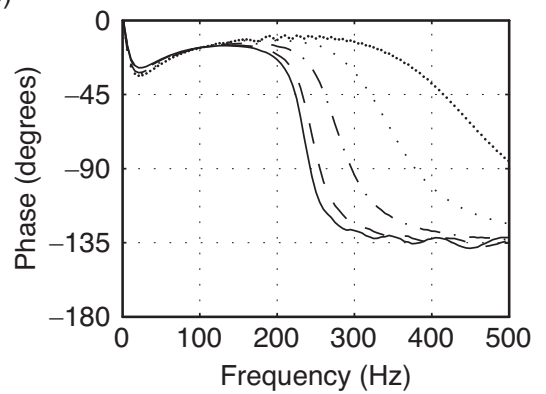

(c)

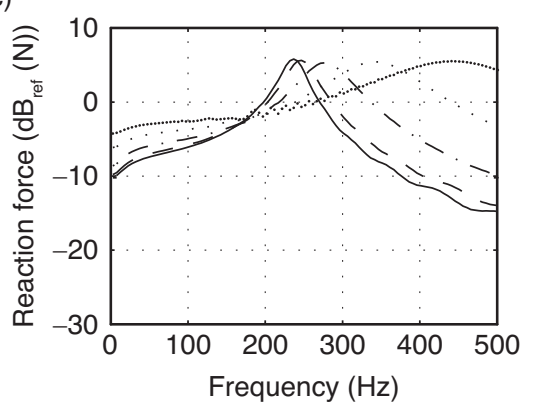

(d)

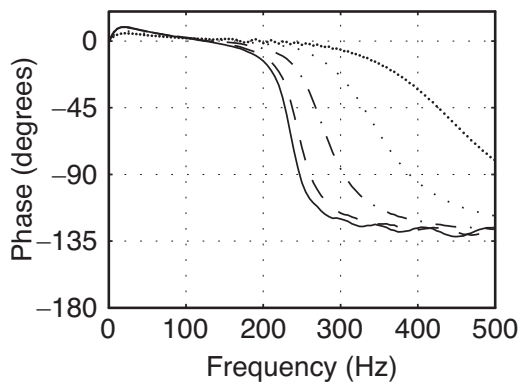

Figure 7. Steady-state magnitude and phase of $(a-b)$ the displacement, $(c-d)$ the reaction force, at a point under the load plotted against excitation frequency, for five preload levels. — $0 \mathrm{kN},---: 25 \mathrm{kN}$, - - -: $50 \mathrm{kN}, \ldots \ldots . . .: 75 \mathrm{kN}$ and ....: $100 \mathrm{kN}$.

amplitudes decrease with increase in preload due to the resulting higher tangent stiffness of the railpads in the vicinity of the load. It can be seen that, for some frequencies, there is a difference of up to $20 \mathrm{~dB}$ between the linear case of $0 \mathrm{kN}$ preload and the strongly nonlinear case of $100 \mathrm{kN}$. The increased stiffness also leads to increase in cut-on frequency from $237 \mathrm{~Hz}$ to $440 \mathrm{~Hz}$ between these preload levels. For the reaction force in Fig. 7(c), as the preload increases, the proportion of the static load that goes into the railpad directly underneath it increases from about $32 \%$ at $25 \mathrm{kN}$ to $42 \%$ at $100 \mathrm{kN}$ (see Table 1 for details). Although the maximum reaction forces at resonance remain fairly constant, the dynamic amplification reduces as preload increases. The dynamic amplification is the ratio of the peak reaction force amplitude at any given frequency to that at $0 \mathrm{~Hz}$. Here also, the disparities between $0 \mathrm{kN}$ and $100 \mathrm{kN}$ loads reach up to $20 \mathrm{~dB}$ at some frequencies.

\subsection{Results for the moving load}

Results will now be presented for the case of a moving harmonic load on the track model. For the following results, the FE model consisted of 80 fastener bays with four $0.15 \mathrm{~m}$ long elements per fastener bay. The time it takes the load to traverse the length of one element is $T_{l}=L / v$, thus the total time for the load to move over the entire track length is $T=N T_{l}$. The time step used in the computation of the track responses, $\Delta t=T_{l} / \kappa$, where $\kappa$ is a positive integer, the value of which is chosen depending on the excitation frequency.

Similar to the stationary load case, the FEM will now be validated against the FTM for the moving load problem using linear parameters. Figure 8 shows the displacements of the rail due to a load moving at $v=250 \mathrm{kmh}^{-1}$ and oscillating at $20 \mathrm{~Hz}$. Comparison is done between the FEM and FTM for both fixed point displacement-time history as in Fig. 8(a) and for that under the moving load as in Fig. 8(b). In both cases the results are in excellent agreement.

Figure 9 shows the static and dynamic displacements of the rail due to a load moving at $v=72 \mathrm{kmh}^{-1}$. The load consists of a static part of $75 \mathrm{kN}$ and a unit amplitude dynamic part oscillating at $10 \mathrm{~Hz}$. Comparison is done between the linear and nonlinear cases. The static displacements shown in Fig 9(a) clearly illustrate the 
(a)

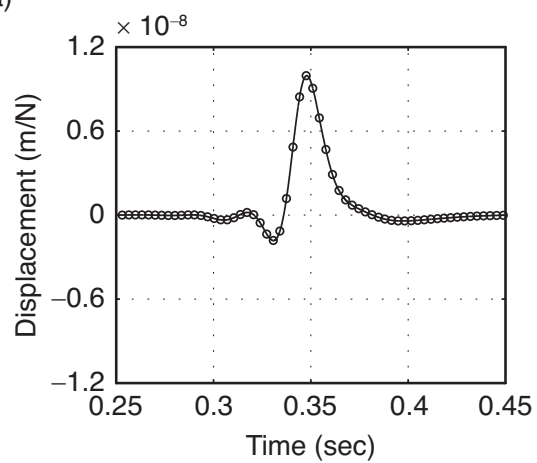

(b)

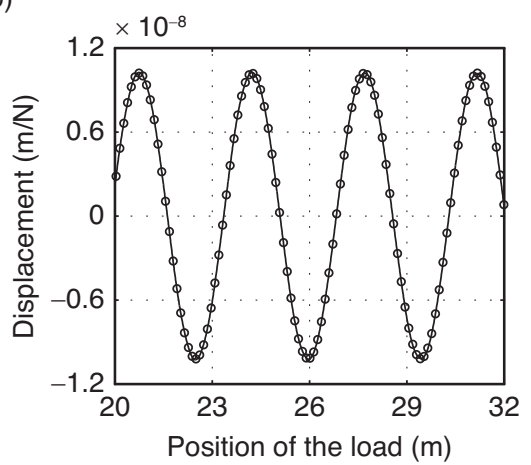

Figure 8. Displacement of the rail due to a load moving at $v=250 \mathrm{kmh}^{-1}$ and oscillating at $20 \mathrm{~Hz}$, (a) at the midpoint and (b) under the load. Comparison between FEM (—) and FTM ( $(\circ)$.

(a)

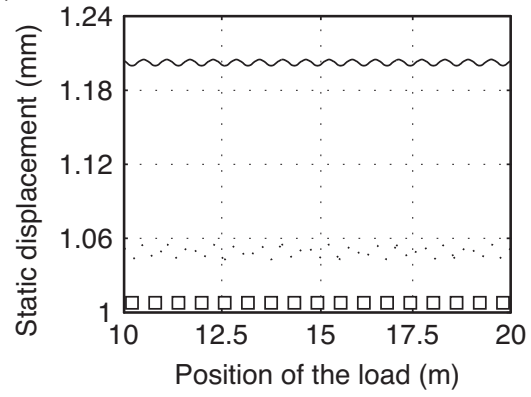

(b)

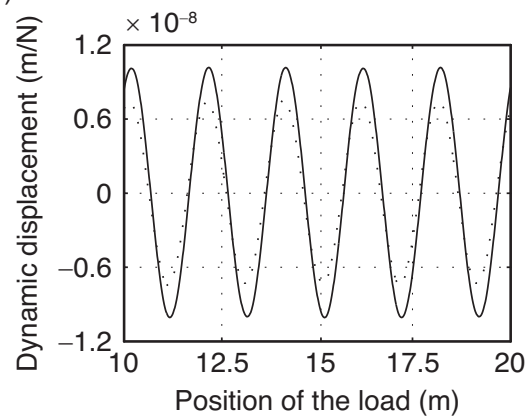

(c)

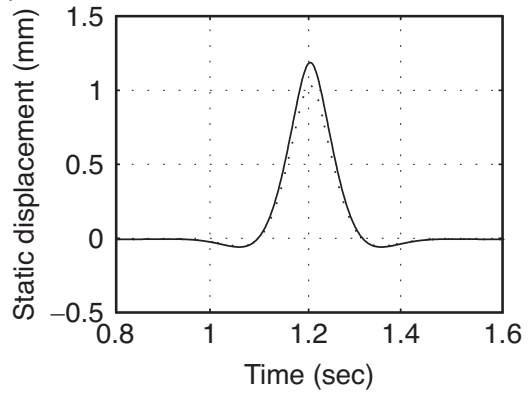

(d)

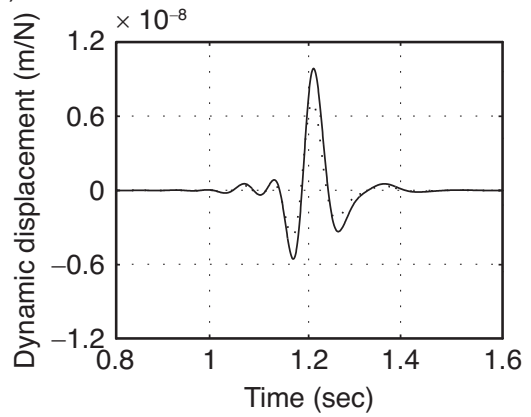

Figure 9. Static and the dynamic displacements of the rail due to a load moving at $v=72 \mathrm{kmh}^{-1}$, with the dynamic part oscillating at $10 \mathrm{~Hz}$. (a) and (b): displacements under the load, (c) and (d): displacements at $x=N L / 2$. Comparison between linear (—) and nonlinear (.........). $\square$ : railpad position.

effect of varying track stiffness caused by the discrete railpad supports. The load exhibits a periodic oscillation with spatial period that is equal to the railpad spacing, $d$, with troughs at the railpad positions (marked by $\square$ ) where the stiffness is greatest and peaks at midspan where it is lowest.

In the nonlinear case, since the static load results in a higher loaded stiffness of the pads close to it, the resulting dynamic displacement in Fig. 9(b) has a much smaller amplitude than that of the linear case. There is also a phase difference between the two responses and this is due to the aforementioned phase difference between the dynamic reaction forces and stiffnesses of the railpads at low frequencies.

In Fig. 9(c) and 9(d), the static and dynamic displacements of the midspan of the track are shown. For the dynamic displacement shown in Fig. 9(d), the effect of accounting for the load rate results in a distortion of the point response, as there is 
(a)

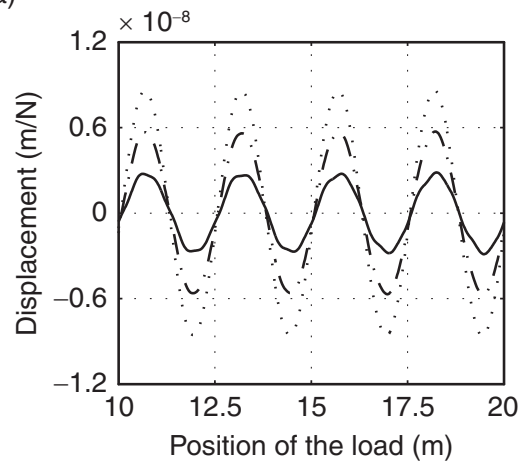

(b)

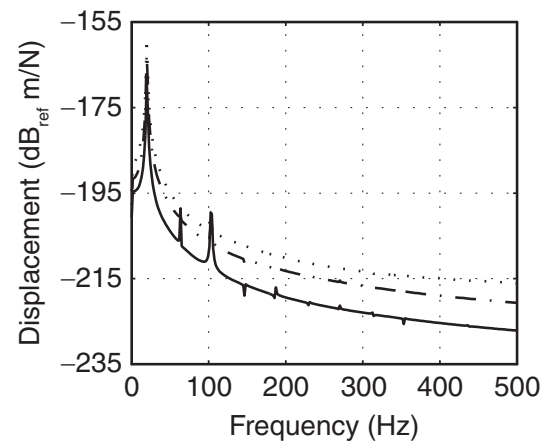

Figure 10. (a) Dynamic displacement response of a point under a load moving at $180 \mathrm{kmh}^{-1}$ and oscillating at $20 \mathrm{~Hz}$, with static component of $40 \mathrm{kN}($......), $75 \mathrm{kN}(-\cdot-)$, and $125 \mathrm{kN}(-)$, (b) Corresponding Fourier magnitude spectrum of the displacements.

no symmetry in the response. Because of the Doppler effect, the point at midspan experiences the load, is oscillating with a higher frequency when approaching its position and a lower frequency when departing.

The effect of magnitude of the static load will now be discussed. Figure 10(a) shows the dynamic displacement under a load moving at $v=180 \mathrm{kmh}^{-1}$ and oscillating at $f_{0}=20 \mathrm{~Hz}$. The results are plotted for static loads of 40, 75 and $125 \mathrm{kN}$, corresponding to light and heavy passenger trains and a freight train respectively. Generally, increasing the static load on the track increases the forces on the railpads and consequently their static stiffnesses. For a stationary static load, the effect of this increase in the stiffness is mainly a reduction in response of the track, since the stiffness distribution under the static load does not change. However, for the case of a moving load, the effects of the change of stiffness from unloaded to loaded and vice versa depends on the level of the static load and the speed at which the load is moving. It should be noted that the length of the loaded zone (i.e. limited to about 8-12 faster bays) does not significantly change with load. Therefore, for a given load speed, the time it takes a railpad to become loaded and unloaded remains fairly constant. For higher preloads, however, the loaded and unloaded displacements vary widely and therefore it changes at a much faster rate than for lower loads. A consequence of this is nonlinear oscillations of the track as demonstrated by the higher static loads. This effect can be accentuated by loads moving with high speeds.

In order to study the frequency content of the dynamic response of the track, the Fourier magnitude spectrum is also presented in Figure 10(b) for all the cases in Fig. 10(a). The spectrum is computed using the Fast Fourier Transform (FFT) procedure.

For all three cases, the main peaks in the response amplitude occur at the excitation frequency of $20 \mathrm{~Hz}$, with smaller peaks occurring at other frequencies. When a static load moves on a discretely supported track, it excites the fastener passing frequency, $f_{f p}=v / d \approx 83 \mathrm{~Hz}$ for the current parameters. For an oscillating moving load, on the other hand, two distinct frequencies are excited (due to Doppler effect), these are: $f_{f p}-f_{0}$ and $f_{f p}+f_{0}$, which results in 63 and $103 \mathrm{~Hz}$, as shown in Fig. 10(b). The severity of these two peaks becomes more pronounced as the preload increases. This is in contrast to what happens at the excitation frequency, where the higher railpad stiffness results in lower response magnitude.

Figure 11 shows the peak (a) displacement and (b) reaction force for the middle railpad, plotted against frequency of oscillation of a load moving at $72 \mathrm{kmh}^{-1}$. In Fig. 11(a), similar behaviour observed for the non-moving load case can also be seen here. This suggests that the effect of convection of the load, at this speed, does not significantly affect the dynamic behaviour. Because of the influence of the moving load, however, the cut-on frequencies are shifted to slightly lower values for all levels of static load. 
(a)

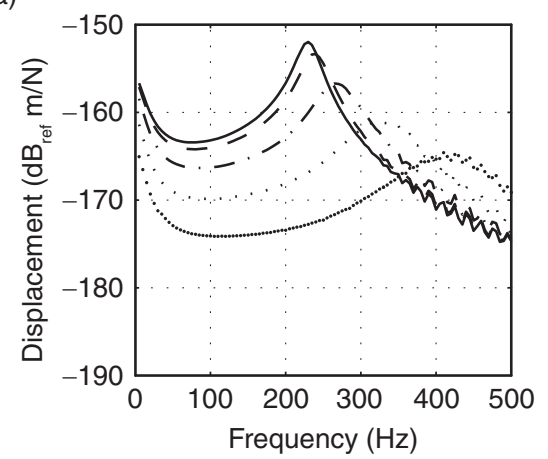

(b)

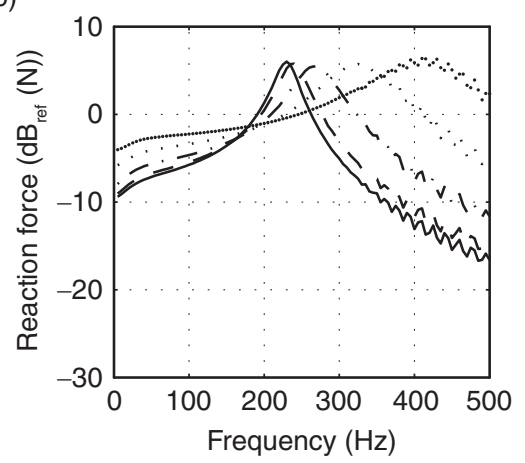

Figure II. Maximum dynamic (a) displacement and (b) reaction force of the railpad under the midpoint of the beam, plotted against oscillating frequency of a load moving at $v=72 \mathrm{kmh}^{-1}$ for five static load levels. Key as for Figure 7.

\section{CONCLUSIONS}

In this paper, the effects of preload and frequency dependent railpad properties on the dynamic behaviour of a railway track have been investigated. A nonlinear railpad model has been developed based on the Poynting-Thomson visco elastic model. This model was then included into a discretely supported track model to study the influence of the railpad model on the track dynamics. The track model, which is formulated using the finite element method, is subjected to combined static and dynamic load and is solved in the time domain using numerical integration. The model is validated, for the linear case, using the Fourier transformation method to the wavenumber-frequency domain.

The key conclusions are summarised as follows:

1. The static preload induces a loaded stiffness distribution of the railpads in its vicinity, due to the nonlinear load-displacement relationship. Depending on its magnitude, the railpads close to the load can become much stiffer, whereas those about 3 4 $\mathrm{m}$ from the load remain unloaded with linear parameters.

2. As the preload increases, the dynamic stiffness increases, resulting in lower displacement amplitudes. For static loads of up to $100 \mathrm{kN}$, the use of a constant linear parameter railpad model results in an overestimation of the track dynamic response amplitudes by up to $20 \mathrm{~dB}$ for some frequencies, compared to the nonlinear model. The opposite effect happens for the reaction forces, which increase with increasing preload.

\section{ACKNOWLEDGEMENT}

The authors wish to acknowledge the funding of this work through the Dean of Engineering Research Scholarship for International Excellence at the University of Nottingham.

\section{REFERENCES}

[1] Grassie SL, Gregory RW, Harrison D and Johhnson KL, The dynamic response of railway track to high frequency vertical excitation. Journal of Mechanical Engineering Science (1982), 24(2), 77-90.

[2] Frýba L, Vibration of solids and structures under moving loads (1999), 3rd edition, Thomas Telford, London.

[3] Mallik AK, Chandra S and Singh AB, Steady-state response of an elastically supported infinite beam to a moving load. Journal of Sound and Vibration (2006), 291, 1148-1169. 
[4] Fenander A, Frequency dependent stiffness and damping of railpads. Proceedings of the Institution of Mechanical Engineers, Part F: Journal of Rail and Rapid Transit (1997), 211, 51-62.

[5] Thompson DJ, van Vliet WJ and Verheij JW, Development of an indirect method for measuring the high frequency dynamic stiffness of resilient elements. Journal of Sound and Vibration (1998), 213(1), 169-188.

[6] De Man AP, Dynatrack: a survey of dynamic railway track properties and their quality (2002), PhD thesis, TU Delft, Delft.

[7] Maes J, Sol H and Guillaume P, Measurements of the dynamic railpad properties. Journal of Sound and Vibration (2006), 293, 557-565.

[8] Harris J and Stevenson A, On the role of nonlinearity in the dynamic behavior of rubber components. Rubber Chemistry and Technology (1986), 59(5), $740-764$.

[9] Fenander A, A fractional derivative railpad model included in a railway track model. Journal of Sound and Vibration (1998), 212(5), 889-903.

[10] Sjöberg MM and Kari L, Non-linear behavior of a rubber isolator system using fractional derivatives. Vehicle System Dynamics (2002), 37(3), 217-236.

[11] Bruni S and Collina A, Modelling the viscoelastic behaviour of elastomeric components: An application to the simulation of train-track interaction. Vehicle System Dynamics (2000), 34(4), 283-301.

[12] Wu TX and Thompson DJ, The effects of local preload on the foundation stiffness and vertical vibration of railway track. Journal of Sound and Vibration (1999), 219(5), 881-904.

[13] Wu TX and Thompson DJ, The vibration behavior of railway track at high frequencies under multiple preloads and wheel interactions. Journal of Acoustical Society America (2000), 108(3):1046-1053.

[14] Kargarnovin MH, Younesian D, Thompson DJ and Jones CJC, Response of beams on nonlinear viscoelastic foundations to harmonic moving loads. Computers \& Structures (2005), 83, 1865-1877.

[15] Nguyen V-H and Duhamel D, Finite element procedures for nonlinear structures in moving coordinates. Part 2: Infinite beam under moving harmonic loads. Computers \& Structures (2008), 86, 2056-2063.

[16] Sato Y, Morimura T and Watanabe S, Theoretical analyses and experimental results on track moduli with use of wheelset drop test. Vehicle System Dynamics (1995), 24(Sup1), 164-179.

[17] Kouroussis G, Verlinden O and Conti C, A two-step time simulation of ground vibrations induced by the railway traffic. Proceedings of the Institution of Mechanical Engineers, Part C: Journal of Mechanical Engineering Science (2012), 226(2), 454-472.

[18] Kouroussis G, Gazetas G, Anastasopoulos I, Conti C and Verlinden O, Discrete modelling of vertical tracksoil coupling for vehicletrack dynamics. Soil Dynamics and Earthquake Engineering (2011), 31(12), 1711-1723. 
[19] Kouroussis G, Verlinden O and Conti C. C, Influence of some vehicle and track parameters on the environmental vibrations induced by railway traffic. Vehicle System Dynamics (2012), 50(4), 619-639.

[20] Uzzal RUA, Ahmed AKW and Bhat RB, Modelling, validation and analysis of a three-dimensional railway vehicle track system model with linear and nonlinear track properties in the presence of wheel flats. Vehicle System Dynamics (2013), 51(11), 1695-1721.

[21] Wu TX and Thompson DJ, The effects of track non-linearity on wheel/rail impact. Proceedings of the Institution of Mechanical Engineers: Journal of Rail and Rapid Transit (2004), 218(Part F), 1-15.

[22] Bathe K-J, Finite element procedures (2006), Prentice Hall, New Jersey.

[23] Bathe K-J and Baig MMI, On a composite implicit time integration procedure for nonlinear dynamics. Computers \& Structures (2005), 83, 2513-2524.

[24] Rieker JR, Lin Y-H and Trethewey M.W, Discretization considerations in moving load finite element beam models. Finite Elements in Analysis and Design (1996), 21(3), 129-144. 